\title{
Sobre-duración: una Aproximación Cuantitativa
}

\author{
Cristian M. Carvajal (1), José A. González (1), Claudia A. Tassara ${ }^{(2)}$ y María S. Álvarez. ${ }^{(2)}$ \\ (1) Universidad de Playa Ancha, Fac. de Ciencias Naturales y Exactas, Laboratorio Experimental de Saberes \\ Matemáticos (LABESAM), Unidad de Soporte Estadístico para la Investigación (USEPI). Avda. Leopoldo Carvallo 270 , \\ Playa Ancha, Valparaíso-Chile. \\ (e-mail: cristian.carvajal@upla.cl). \\ (2) Universidad de Playa Ancha, Unidad de Análisis Institucional, Vicerrectoría de Desarrollo, Avda. Playa Ancha 850, \\ Valparaíso-Chile.
}

Recibido Sep. 15, 2017; Aceptado Nov. 23, 2017; Versión final Dic. 18, 2017, Publicado Jun. 2018

\begin{abstract}
Resumen
Se presenta un estudio sobre la titulación oportuna, un problema de gran interés para las instituciones de educación superior. En el proceso de organizar la investigación, se toma como concepto basal lo denominado sobre-duración, en donde la titulación oportuna se transforma en un caso particular. Se propone un modelo predictivo para la sobre-duración aplicado a un conjunto real de datos de una institución de educación superior. Se establecen los niveles de significancia de las variables caracterizadas como predictoras, además de variaciones porcentuales por unidad de cambio en ellas. Para facilitar el estudio de la dependencia jerárquica, se estructura una metodología de representación denominada raíz jerárquica de tercer orden, que permite cuantificar los efectos y su nivel de implicancia. Como consecuencia de esto se pudo establecer que la sobre-duración puede ser explicada por un conjunto de variables de primer orden, tales como género, año de egreso de la enseñanza media, grupo de dependencia del colegio, promoción, cargo del profesor guía de tesis, entre otras, de las cuales muchas de ellas no pueden ser perturbadas de forma directa.
\end{abstract}

Palabras clave: titulación oportuna; titulación retardada; educación superior; sobre-duración

\section{Over-duration: A Quantitative Approach}

\begin{abstract}
A study on timely qualification is presented, a problem of great interest for higher education institutions. In the process of organizing research, the so-called over-duration is taken as the baseline concept, where the appropriate degree is transformed into a particular case. A predictive model for over-duration applied to a real data set of a higher education institution is determined. The levels of significance of the variables characterized as predictors are established, as well as percentage variations per unit of change in them. To facilitate the study of hierarchical dependence, a representation methodology called the hierarchical root of the third order is structured, which allows to quantify the effects and their level of implication. As a consequence of this it was established that the over-duration can be explained by a set of variables of first order, such as gender, year of graduation from high school, college dependency group, promotion, position of the teacher thesis guide, among others, of which many of them cannot be directly disturbed.
\end{abstract}

Keywords: timely graduation; late graduation; higher education; over-duration 


\section{INTRODUCCIÓN}

En el último decenio el número de estudiantes matriculados en la educación superior chilena ha mostrado un crecimiento sostenido, alcanzando una matrícula total al año 2016 de 1.178.437 estudiantes, lo que representa un aumento del 57,7\%, estadísticas recogidas por el Servicio de Información de Educación Superior (SIES, 2016). Sin embargo, estas cifras y su impacto, se ven reducidas si no se tiene a la vista un conjunto de otros indicadores que permita tener una visión más amplia y aguda de los derroteros seguidos por la educación superior chilena, tales como, las tasas de deserción y las tasas de titulación oportuna, pues una política que se enfoque exclusivamente en aumentar los porcentajes de ingresos y descuide estos otros elementos fundamentales, es una política truncada, poco eficaz y generadora de una sensación de frustración en los estudiantes que ingresan a la educación superior y que ven disminuidas sus posibilidades de finalizar a tiempo y con éxito sus estudios, pues la sociedad chilena sigue viendo en la educación el camino más seguro hacia una vida de mayor prosperidad (OCDE, 2009).

Es por lo anterior, que el presente trabajo centrará sus esfuerzos en estudiar y analizar de manera general estos fenómenos y de forma particular la titulación oportuna, para ello se ha considerado el concepto sobreduración, definido como el cociente entre 1) la diferencia entre el número de semestres que demoran en obtener su título los estudiantes y el número de semestres esperados de la carrera y/o programa que cursaron, y 2) el número esperado de semestres que dura su carrera y/o programa (SIES,2016), en este sentido el artículo se ha propuesto como objetivo general: Determinar y validar un modelo predictivo para la sobreduración, en los estudiantes de pregrado de la Universidad de Playa Ancha de Ciencias de la Educación y como objetivos específicos: (i) Determinar la significancia de las variables predictoras, (ii) Cuantificar los incrementos o efectos sobre el tiempo de titulación por variaciones unitarias en los predictores significativos, (iii) Estructurar un modelo parsimonioso en la predicción o explicación del tiempo de sobre-duración y (iv) Cuantificar el poder predictivo del modelo a proponer. Para alcanzar estos objetivos y considerando la característica métrica de la variable respuesta (tiempo en años de sobre-duración), se considera en el estudio una variable con distribución Poisson.

Este artículo analiza los datos de los titulados, entre los años 2011-2015, de carreras profesionales de la Universidad de Playa Ancha de Ciencias de la Educación, para las cohortes 2006-2011, obteniendo un tamaño muestral de 1179 alumnos con un nivel de confianza del 95\%. Por otro lado, con el propósito de implementar una metodología organizacional de las variables predictoras, el presente trabajo propone una estructura denominada "Raíz Jerárquica de Tercer Orden para el Tiempo de Sobre-duración", esta estructuración jerárquica permite especificar las variables predictoras de efectos directos e indirectos que intervienen en el fenómeno de la sobre-duración, además, permite cuantificar el impacto de estos predictores sobre la variable respuesta original y finalmente, esta estructura facilita la visualización de los grados de dependencias del conjunto de predictores (Carvajal et al., 2018).

Con respecto al tiempo que demoran los estudiantes en obtener la titulación en el Sistema de Educación Superior Chileno, el Servicio de Información de Educación Superior (SIES, 2016) estableció que, para las carreras profesionales sin licenciatura, el tiempo medio es de 10.6 semestres en los institutos profesionales y 11 semestres en las universidades, finalmente la duración media real de las carreras profesionales con licenciatura es de 13.4 semestres. Por otro lado, si se analiza la diferencia entre el tiempo de duración formal de titulación y el tiempo real en las universidades pertenecientes al Consejo de Rectores de Universidades Chilenas ( $\mathrm{CRUCH}$ ) y las que no pertenecen, las primeras tienen un incremento de un $38 \%$ de la duración real respecto a la formal, mientras que para las segundas este incremento alcanza un 31\% (Pey etal., 2012). Pero ¿Cuáles son los efectos de este fenómeno?, el informe sobre Educación Superior en América Latina y el Caribe (IESALC, 2000-2005), sostiene que los efectos negativos del rezago en la titulación, repercute al menos en tres dimensiones: la dimensión institucional, la cual se ve coartada de cumplir su misión institucional afectando de manera importante los índices de eficiencia y calidad, la dimensión personal, en donde la sobreduración, en muchos casos redunda en una sensación de frustración y fracaso del estudiante, finalmente la dimensión económica, pues se estima que el rezago universitario conlleva un elevado costo económico para el estudiante y su familia. Es por esto, que contar con evidencia científica, que nos permita entender de mejor manera este fenómeno se hace cada vez más relevante.

\section{MARCO TEÓRICO}

En Chile las investigaciones destinadas a estudiar y analizar la temporalidad en la titulación universitaria, las tasas de graduación y el fenómeno de la deserción en el sistema de educación superior han sido escasas y destinadas principalmente a describir la magnitud de estos fenómenos (Himmel, 2002), a pesar de esto, y mandatado por el Consejo de Rectores de Universidades Chilenas ( $\mathrm{CRUCH})$, se desarrolló una de las investigaciones más completas destinada a comprender el fenómeno de la excesiva duración de las carreras de pregrado en las universidades chilenas pertenecientes al $\mathrm{CRUCH}$, leitmotiv del presente estudio. 
Las instituciones de educación superior denominadas "complejas", tipificación asignada a aquellas universidades acreditadas en docencia, investigación y vinculación con el medio, son las que presentan una mayor diferencia entre el tiempo real de titulación y el tiempo formal de esta, lo cual, puede ser explicado por la convergencia de al menos tres factores, a saber: los procesos de titulación en estas instituciones son más complejos, los estándares de aprobación de los cursos son mayores y el tiempo destinado a la nivelación de sus estudiantes en las competencias básicas son más extensos (Pey et al., 2012). Por otro lado, al explorar las causales que explican la demora en el tiempo de titulación, emergen al menos tres elementos transversales. Un primer elemento dice relación con la estructuración de los procesos formativos, pues un número importante de programas de estudios han sido estructurados pensando en estudiantes que cuentan con una dedicación exclusiva en sus estudios, sin embargo, en el total del sistema universitario hay un $24,24 \%$ de alumnos que trabajan, lo que redunda en una alta carga académica, por otro lado, un segundo elemento fundamental en el retraso de la titulación es el relacionado con el financiamiento, pues cerca de un tercio de los recursos que ingresan a las arcas de las universidades pertenecientes al CRUCH provienen de las familias, situación que repercute en una importante carga financiera que deben sostener los estudiantes y finalmente una excesiva y compleja burocracia, evidenciada en la extensión y dificultad de los hitos que conforman el proceso de titulación (Pey et al., 2012).

El estudio de (Giovagnoli, 2001), ha mostrado que el nivel educacional de los padres influye de manera significativa en la probabilidad de graduarse, en este sentido un estudiante cuyo padre tiene sólo educación básica incompleta, presente una probabilidad de finalizar con éxito sus estudios un $70 \%$ menor que un alumno con un padre profesional. Otros factores condicionantes de la sobre-duración mencionados por (Vera et al., 2012) están relacionado con factores personales, académicos y sociodemográficos, tales como el género, el promedio de la educación básica, el puntaje de admisión de ingreso a la universidad, la situación laboral y factores de carácter institucional, como el currículo y su administración, la conducta de los estudiantes en la selección de los cursos, la orientación hacia el estudiante y la percepción del apoyo recibido por la universidad, en este sentido la orientación por parte del profesor y por parte del coordinador del programa hacia los estudiantes aparece como una variable importante.

El trabajo de (Hammond, 2016), sostiene que los factores que intervienen en la deserción y/o en la sobreduración universitaria, pueden ser configurados en tres categorías analíticas, a saber: factores contextuales, de entre los cuales el autor rescata las representaciones sociales y la asimetría entre el nivel secundario y el nivel terciario; factores organizacionales tales como, la relación de los estudiantes con el cuerpo académico, la infraestructura institucional, la oferta académica y la representación sobre la carrera y finalmente los factores personales, de entre los cuales se destacan la definición vocacional del alumno, la compatibilidad entre el estudio y el trabajo, la situación económica, el contexto familiar, las actividades extracurriculares en la que participa el estudiante, el cumplimiento de metas académicas, las características personales del alumno y su visión profesional, todo lo anterior permite establecer, según el autor, que aquellos estudiantes que estén afectados por algunos de estos factores, se encontrarán en condiciones de vulnerabilidad y por ende, las posibilidades de sobre-duración de sus estudios o del abandono del mismo, se verán incrementadas de manera significativa. Otro elemento significativo a tener en cuenta al momento de estudiar el fenómeno de la sobre-duración, es el rendimiento académico, en este sentido se puede advertir que el rendimiento académico en estudiantes de educación superior constituye un factor imprescindible y fundamental en la valoración de la calidad educativa y la eficiencia de la enseñanza superior (Garbanzo y Guiselle, 2007). Lo anterior permite establecer una relación virtuosa entre una buena performance académica y una reducción en el tiempo de sobre-duración.

Desde una mirada productiva, se puede establecer que el rendimiento académico del estudiante está directamente relacionado con un conjunto de insumos que este dispone, los cuales pueden ser externos o internos, lo que la literatura ha denominado función de producción y lo que trata de hacer es modelar el producto educativo como el resultado de una función que utiliza insumos (Di Gresia, 2007) luego lo importante es la calidad del producto elaborado. En términos de las variables que intervienen significativamente en el rendimiento académico, las investigaciones (Garbanzo y Guiselle, 2007; González, 2017; Rodríguez, Fita y Torrado, 2004; Di Gresia, 2007 y Oriol et al., 2017) han agrupado estas en cuatro dimensiones a describir; la dimensión personal cuyos factores asociados son entre otros, las competencias cognitivas, la motivación, el auto concepto académico, la autoeficacia percibida, el bienestar psicológico, sexo, asistencia a clases, formación académica preuniversitaria y nota de acceso a la universidad; una segunda dimensión es la social, la que cuenta con los factores referidos a las diferencias sociales, al entorno familiar, al nivel educativo de los padres, al contexto socioeconómico y a las variables demográficas; finalmente la dimensión institucional, cuyos factores se relacionan con la complejidad de los estudios, las condiciones institucionales, los servicios de apoyos institucionales, ambiente estudiantil, relación estudiante-profesor y pruebas específicas de ingreso a la carrera. Todas estas variables se encuentran presente de manera directa o indirecta en los fenómenos de deserción y sobre-duración, lo que permite suponer una relación entre estas y el rendimiento académico. 
Finalmente, y desde una perspectiva de responsabilidad social universitaria (Schmelkes, 2001) establece que una educación de calidad tiene que ser eficaz, es decir, que se logren los objetivos propuestos para todos los alumnos, además esta debe ser eficiente, es decir, que se atienda con responsabilidad la relación existente entre los resultados obtenidos y los costos destinados a obtenerlos, lo anterior pone de manifiesto la importancia que tiene el establecer políticas educativas destinadas a maximizar las tasas de titulación oportuna, pues no basta con retener al estudiante, es también necesario optimizar los tiempos de titulación.

\section{METODOLOGÍA}

El presente estudio está enmarcado en un paradigma positivista, con alcance cuantitativo, de medición longitudinal, en un contexto propositivo (Hernández et al., 2014), el cual incorpora un conjunto de relaciones entre preguntas de investigación e hipótesis de trabajo, a saber:

Tabla 1: Relaciones entre preguntas de investigación e hipótesis de trabajo

Preguntas de investigación

¿Qué variables académicas afectan de manera significativa la sobre-duración?
Hipótesis de trabajo (significance level 5\%).

La especialidad de la carrera afecta significativamente en la sobre-duración.

El grado académico del director de tesis afecta significativamente en el tiempo de sobre-duración.

Las responsabilidades administrativas del director de tesis afecta significativamente en el tiempo de sobre-duración.

El año de egreso de enseñanza media afecta significativamente en el tiempo de sobre-duración.

¿La sobre-duración es un problema de efecto homogéneo o caracteriza algunas facultades y/o carreras?

Las facultades de salud e ingenería presentan diferencias significativas en el tiempo de sobre-duración.

La condición de trabajo al inicio del periodo académico es una causal significativa en la sobre-duración.

¿Las responsabilidades familiares y de contexto no académico explican de manera significativa la sobreduración?

El nivel educacional de los padres afecta significativamente el tiempo de sobre-duración.

La condición socio-económica asociada al quintil más bajo afecta significativamente en el tiempo de sobre-duración.

¿La sobre-duración es una cuestión de género?
No existen diferencias significativas entre los efectos del género como predictor de la sobre-duración.

\section{Unidad de análisis, universo y muestra}

El universo de estudio en la investigación son los titulados en los años 2011-2015 de las carreras profesionales de la Universidad de Playa Ancha de Ciencias de la Educación para las cohortes 2006-2011, correspondiente a 3484 titulados. Se debe indicar la presencia de cluster naturales como promociones, carreras y facultades, los cuales dieron los lineamientos para las comparaciones, las unidades de estudio son realizaciones de procesos probabilísticos, por lo tanto, hay presencia de aleatoriedad. El tamaño de la muestra fue de 1179 a razón de registros incompletos y datos perdidos, este tamaño no ha afectado el nivel de confianza del 95\%, y error de estimación del $\pm 5 \%$, según la fórmula de Namakforoosh, pues se supera el $25 \%$ de representatividad.

Para la recolección de datos, se utilizó la información proporcionada por la Unidad de Análisis Institucional (UAI) perteneciente a la Vicerrectoría de Desarrollo, cuyas fuentes son la Oficina de Títulos y Grados, Unidad de Admisión y Selección, Dirección de Desarrollo Estudiantil y Dirección General de Pregrado, todas ellas pertenecientes a la Universidad de Playa Ancha de Ciencias de la Educación. Estos datos fueron sometidos a un proceso de validación basado en el índice de validez $\Gamma$ definido por [1+(n-1) $\left.\rho_{x x}\right] / n$ (González et al., 2016). Por su parte la manipulación de los datos, en el proceso de transformación a información, se realizó utilizando el software r-project 3.3.3., siendo este de carácter libre.

\section{Técnica de análisis de datos}

El análisis de datos fue realizado de forma incremental, siguiendo tres etapas: i) etapa correlacional; ii) etapa Inferencial; y iii) Ajuste del Modelo: 
Etapa correlacional: se aplicaron pruebas correlacionales, cuyo criterio de decisión fue el p-valor, en un nivel de significancia de 5\%, las técnicas utilizadas dependieron del nivel de medición de las variables que se sometieron a correlación (Hernández et al., 2014). Por ejemplo, Chi cuadrada para variables nominales; el coeficiente de rangos ordenados de Spearman para variables ordinales; o el coeficiente de correlación de Pearson para variables de intervalos o de razón. La etapa correlacional buscó identificar la anidación de variables o variables de efectos indirectos.

Etapa Inferencial: se aplicaron pruebas de contrastes de medias para poder asumir igualdad de estratos, todas basadas en el criterio de decisión del p-valor y considerando un nivel de significancia del $5 \%$.

Ajuste del Modelo: se propone un modelo explicativo, indicando variables causales de primer, segundo y tercer orden, procurando un modelo parsimonioso. Todas las variables constituyentes del modelo están a un nivel de significancia del $5 \%$.

La presentación de los resultados se basa en una raíz jerárquica de tercer orden, metodología propuesta por los autores para facilitar la visualización de los grados de dependencia de un conjunto de predictores a partir de una variable respuesta, esta estructuración permite especificar las variables de efectos directos e indirectos, además de poder cuantificar el impacto sobre la variable de respuesta original. Cuando una metodología de estructuración jerárquica es aplicada en la explicación de la variable sobre-duración, es posible identificar las variables que le competen, por ejemplo, a la institución formadora, o identificar sobre cuales variables es posible intervenir, por lo tanto, optimizar el uso de recursos o esfuerzos. El procedimiento de análisis de la raíz jerárquica en términos de significancia es el siguiente: señalar que la variable afecta significativamente en segundo orden a la sobre-duración, implica que afecta significativamente a una variable de primer orden, de manera similar una de tercero sobre segundo orden.

\section{RESULTADOS Y DISCUSIÓN}

Los resultados son presentados en consistencia con la construcción de la raíz jerárquica de tercer orden, para ello fueron desarrolladas cada una de las etapas antes descritas, previa agrupación de las variables en cuatro dimensiones. Los resultados se organizarán en tres niveles caracterizados por el orden jerárquico que ocupan los predictores.

\section{Análisis de las variables de Primer Orden}

En esta sección se explicitan los efectos y significatividad de las variables predictoras, teniendo en consideración que la variable de respuesta es de conteo (Poisson process). Son identificadas las variables sexo, año de egreso de enseñanza media, grupo de dependencia del colegio, promoción, duración desde el ingreso hasta el egreso de la universidad, tiempo entre el egreso de la universidad y defensa de la tesis, tiempo entre el año de defensa y el año del pago del título, duración de la carrera en semestres y el cargo del profesor guía de tesis, como las variables predictoras de primer orden, de las cuales sexo, año de egreso de enseñanza media, grupo de dependencia del colegio, pertenecen a la primera dimensión, la promoción pertenece a la segunda, duración desde el ingreso hasta el egreso de la universidad, tiempo entre el egreso de la universidad y defensa de la tesis, tiempo entre el año de defensa y el año del pago del título a la tercera y finalmente, la duración de la carrera en semestres y el cargo del profesor guía de tesis a la cuarta dimensión.

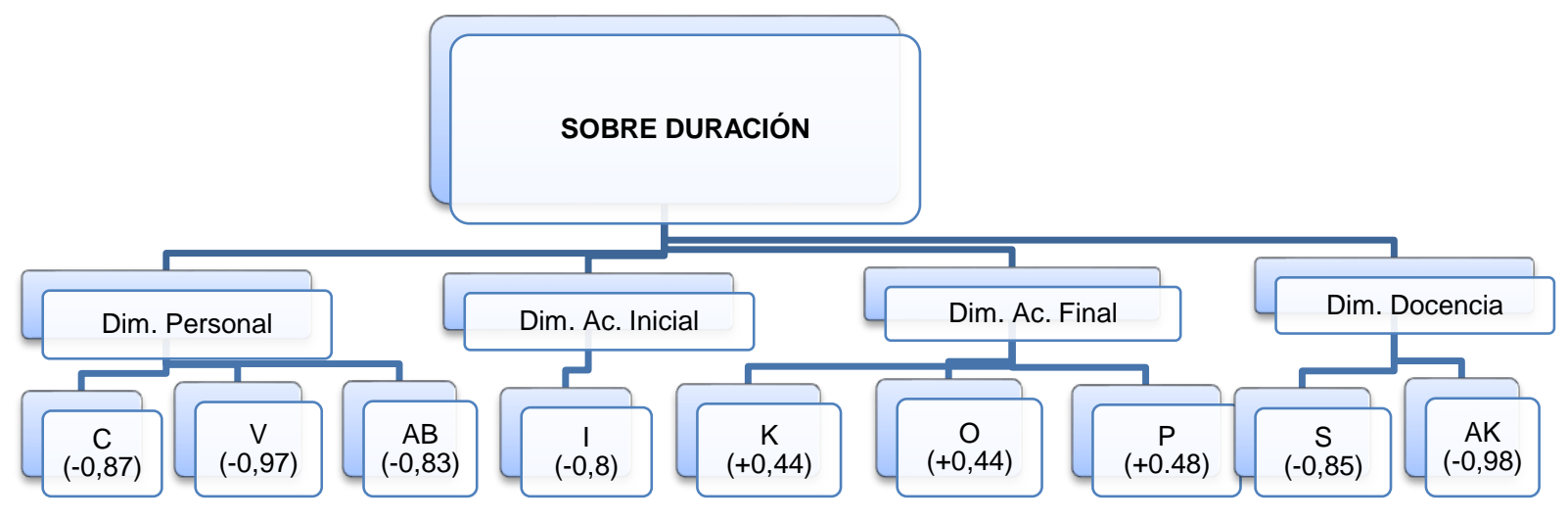

Fig.1: Variables de Primer Orden. 
Tabla 2: Notación de Variables

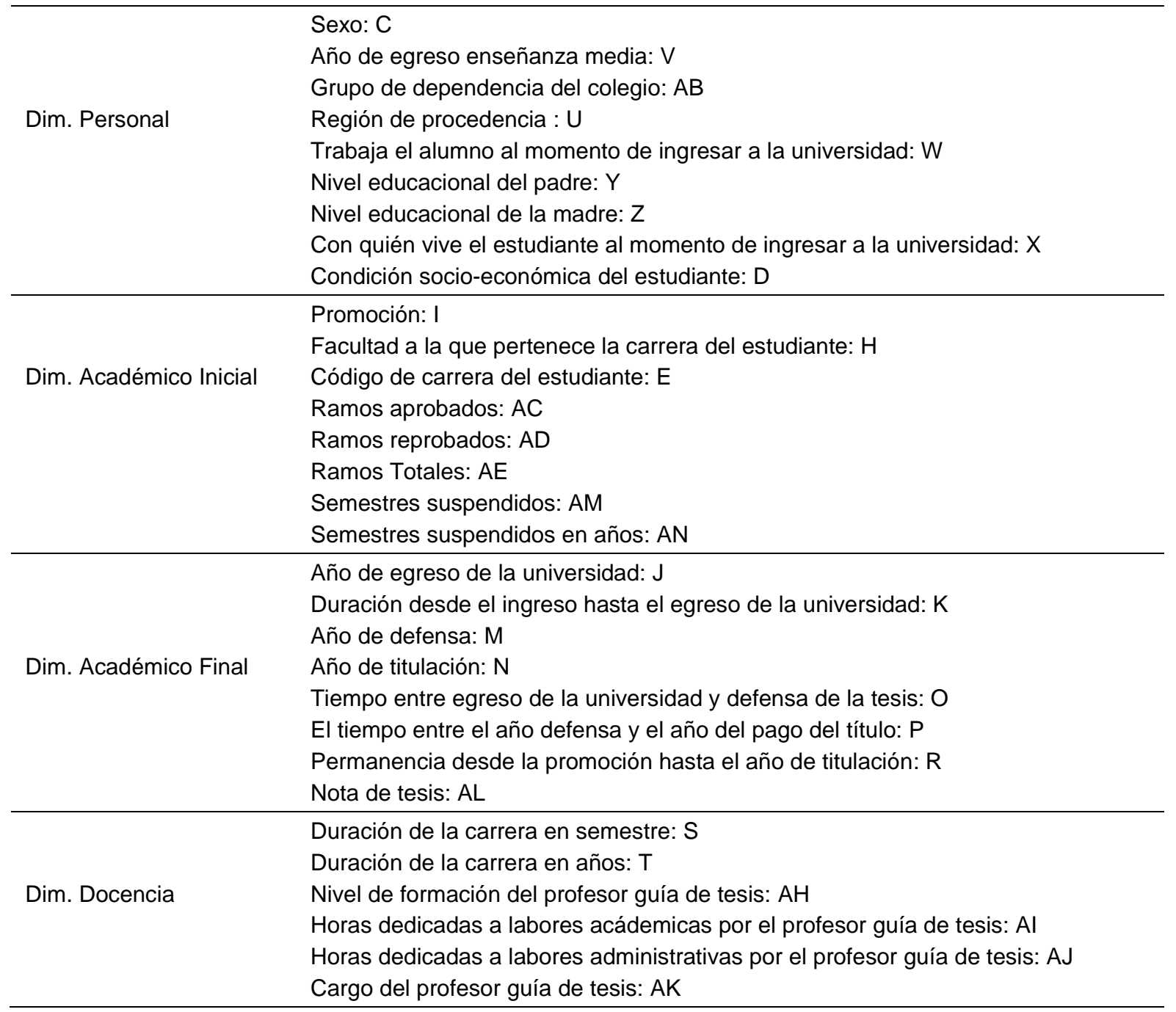

Con respecto al efecto de cada variable predictora de primer orden se indica que, la duración desde el ingreso hasta el egreso de la universidad, el tiempo entre egreso de la universidad y defensa de la tesis y el tiempo entre el año de defensa y el año del pago del título, presentan efectos de tipo positivo sobre la respuesta, esto significa que incrementos unitarios en estas variables se traducen en un aumento en el valor esperado o tiempo esperado de sobre-duración. En el caso de los predictores sexo, año de egreso enseñanza media, grupo de dependencia del colegio, promoción, duración de la carrera en semestres y cargo del profesor guía de tesis, los efectos son negativos, es decir, que incrementos unitarios en estas variables se traducen en una disminución en el tiempo esperado de sobre-duración. Por ejemplo, si consideramos la variable grupo de dependencia del colegio, el cambio de municipal a particular pagado, se traduce en una disminución del tiempo esperado de sobre-duración.

En términos de la cuantificación del impacto de cada uno de los predictores identificados como significativos, la raíz jerárquica, nos permite identificar cuanto incrementa o disminuye, según sea el signo del tiempo esperado de sobre-duración. En función del ejemplo anterior, el efecto de dicha variable es de 0.83 años, es decir, variaciones unitarias en el grupo de dependencia del colegio se traducen en diferencias de casi un año en los tiempos esperados de sobre-duración, esto es, el cambio de municipal a particular pagado, implicaría una disminución de aproximadamente un año en el tiempo esperado de sobre-duración.

\section{Análisis de las variables de Segundo Orden}

Son identificadas la región de procedencia, trabaja el alumno al momento de ingresar a la universidad, nivel educacional del padre, nivel educacional de la madre, facultad a la que pertenece la carrera del estudiante, duración de la carrera en años, nivel de formación del profesor guía de tesis, horas dedicadas a labores académicas por el profesor guía de tesis, horas dedicadas a labores administrativas por el profesor guía de tesis como las variables predictoras de segundo orden. 


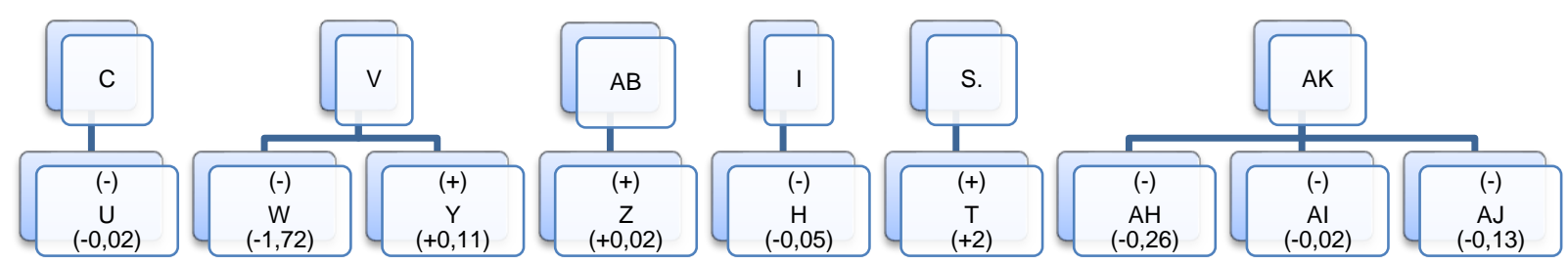

Fig.2: Variables de Segundo Orden

La especificación de las redes para las variables de segundo orden está basada en un modelo lineal generalizado, en este caso particular modelo de regresión lineal múltiple, ya que no se tiene la restricción de conteo en las respuestas. En la Fig. 2, se visualiza la utilización de la simbología (+) y (-), lo que hace referencia al efecto que tiene sobre cada variable predictora, ahora asumida como respuesta. Por ejemplo, la variable trabaja el alumno al momento de ingresar a la universidad esta acompañada del signo (-), en relación al año de egreso enseñanza media, esto significa que incrementos en la variable predictora se traduce en una disminución en el año de egreso enseñanza media. De manera similar para el nivel educacional de la madre acompañado del signo (+), en relación al grupo de dependencia del colegio, significa que incrementos en el nivel educacional de la madre se tiene incrementos en el grupo de dependencia del colegio. Finalmente, el valor asociado a las variables en la Fig.2, establece el efecto que tiene cada predictor sobre la respuesta (en este nivel las respuestas son las variables de primer orden), por ejemplo, la variable nivel de formación del profesor guía de tesis, tiene un efecto negativo de -0.26 sobre la variable cargo del profesor guía de tesis, lo que significa, que aumentar en una unidad el nivel de formación del profesor guía de tesis, afecta inversamente al cargo del profesor guía de tesis, contextualizando, mientras menor es el grado del profesor guía de tesis, hay tendencia a un mayor cargo directivo.

\section{Análisis de las variables de Tercer Orden}

Son identificadas, con quién vive el estudiante al momento de ingresar a la universidad, región de procedencia, nivel educacional de la madre, condición socio-económica del estudiante, código de carrera del estudiante, nivel de formación del profesor guía de tesis las variables predictoras de tercer orden.
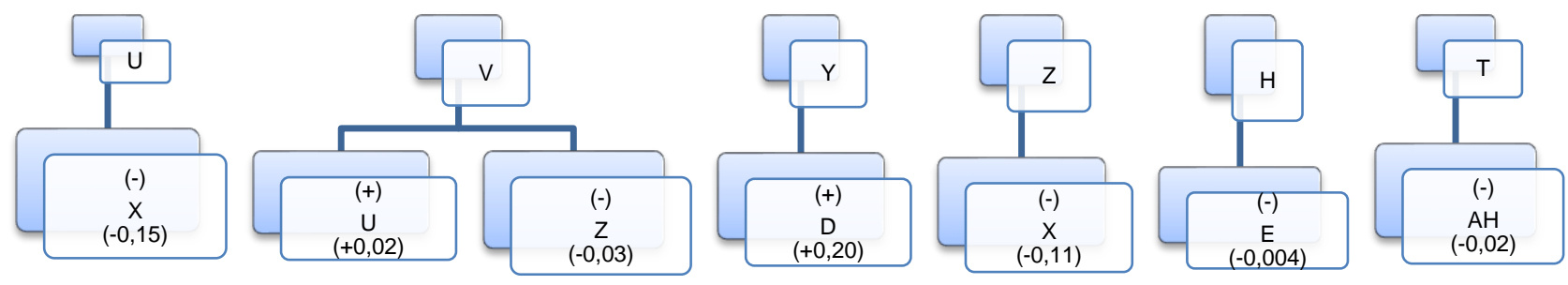

Fig.3: Variables de Tercer Orden.

El análisis de las variables de tercer orden siguen la misma dinámica del análisis anterior, en este sentido podemos observar que en la Fig.3 el predictor región de procedencia tiene asociado un signo positivo (+) en relación a la respuesta año de egreso enseñanza media, luego si aumentamos la región de procedencia, esto redundará en un aumento en la variable año de egreso enseñanza media, con respecto al efecto particular de los predictores se puede establecer, por ejemplo, que el aumento en una unidad de la variable condición socio-económica del estudiante, se traducirá en el aumento medio de 0.20 años en la variable nivel educacional del padre.

\section{Raíz jerárquica de tercer orden para la sobre-duración}

En función de cada una de las etapas antes descritas, se presenta la raíz jerárquica de tercer orden para la sobre-duración. En esta raíz jerárquica, podemos observar las relaciones existentes en los diferentes niveles entre los predictores y sus respuestas, por ejemplo, en el tercer orden la variable condición socioecionómica tiene un efecto positivo sobre el nivel educacional del padre y ésta a su vez tiene un efecto positivo sobre el año de egreso de enseñanza media, lo que redundaría en una disminución de 0.97 años la sobre-duración.

En función del contexto, la investigación incorporó una relación entre preguntas e hipótesis de trabajo (ver tabla 1), de las cuales se concluyó, con un nivel de significancia del $5 \%$, lo siguiente: en cuanto a la pregunta 
¿Qué variables académicas afectan de manera significativa la sobre-duración? Se pudo establecer que una de estas variables es la promoción, la cual, permitió visualizar que las promociones más nuevas disminuyen el tiempo de sobre-duración en aproximadamente 0.8 años; otra variable es la permanencia del estudiante, cuya significatividad se debe a la dependencia lineal en la generación de la variable sobre-duración; la variable duración de la carrera en semestres, las carreras que duran más semestres, disminuye el tiempo de sobreduración en aproximadamente 0.85 años, y por último, la variable cargo del profesor guía de tesis, nos indica que mientras menos cargo tiene el profesor guía, disminuye el tiempo de sobre-duración en aproximadamente 0.98 años. En relación a esta pregunta las hipótesis fueron: (1) La especialidad de la carrera afecta significativamente en la sobre-duración, esta hipótesis no se rechaza en segundo orden. (2) El grado académico del director de tesis afecta significativamente en la sobre-duración, esta hipótesis no es rechazada en segundo orden. (3) Las responsabilidades administrativas del director de tesis afecta significativamente a la sobre-duración, hipótesis no rechazada en primer orden. (4) El año de egreso de enseñanza media afecta significativamente en la sobre-duración, hipótesis no rechazada en primer orden.

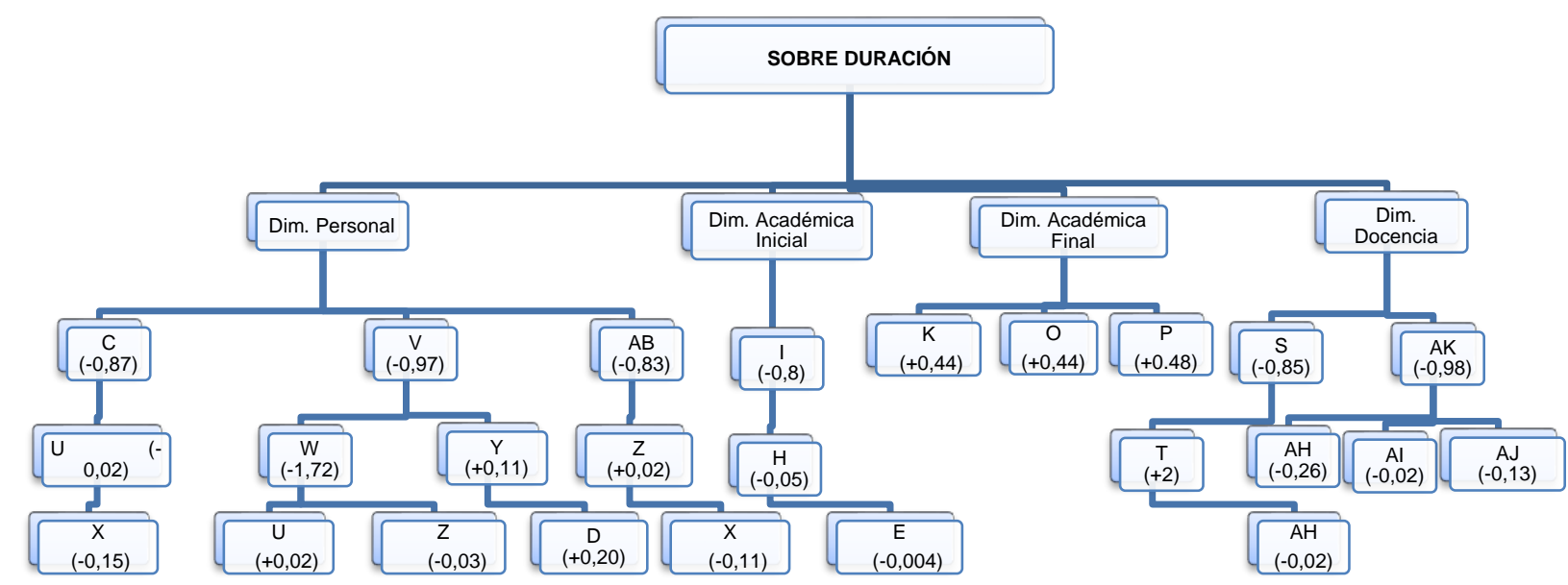

Fig.4: Raíz jerárquica de tercer orden

En cuanto a la pregunta ¿La sobre-duración es un problema de efecto homogéneo o caracteriza algunas facultades y/o carreras? Se pudo establecer que las facultades o carreras no generan efectos significativos en la sobre-duración, sin embargo, se debe indicar que es caracterizada como una variable de influencia en segundo orden sobre la variable promoción. Esto nos permite establecer que la sobre-duración es un problema de efecto homogéneo. En relación a esta pregunta la hipótesis fue: las facultades de salud e ingeniería presentan diferencias significativas en el tiempo de titulación, esta hipótesis es rechazada, ya que no existen diferencias significativas.

En cuanto a la pregunta ¿Las responsabilidades familiares y de contexto no académico explican de manera significativa la sobre-duración? Se logró establecer la existencia de tres variables predictoras identificadas con efectos significativos, estas son: sexo, año de egreso de enseñanza media y el grupo de dependencia del establecimiento. En relación a esta pregunta las hipótesis fueron: (1) La condición de trabajo al ingreso del periodo académico es una causal significativa en la sobre-duración, hipótesis no rechazada en segundo orden. (2) El nivel educacional de los padres afecta significativamente el tiempo de sobre-duración, esta hipótesis no es rechazada en segundo orden. (3) La condición socio-económica asociada al quintil más bajo afecta significativamente el tiempo de sobre-duración, esta hipótesis no es rechazada en tercer orden. Finalmente en relación a la pregunta ¿La sobre-duración es cuestión de género? La variable sexo es identificada como significativa, favoreciendo a las mujeres en periodos de sobre-duración menores, con diferencias de aproximadamente un año. En relación a esta pregunta la hipótesis fue: no existen diferencias significativas entre los efectos del género como predictor de la sobre-duración, esta hipótesis es rechazada.

\section{EL MODELO}

Esta sección es caracterizada por dos etapas, la primera consitió en identificar las variables de efecto significativo en la explicación de la sobre-duración y la segunda etapa consistió en establecer el poder predictivo del modelo. Las variables que caracterizan cada etapa son distintas, pues las intensiones del modelo son diferentes, esto es, en la primera etapa la intensión es reaccionar como institución sobre aquellos elementos que en funcionamiento están afectando la sobre-duración, en cambio, en la segunda etapa interesa identificar las condiciones de entrada del alumno, de tal manera de poder anticipar procedimientos, en función de los estados de las variables de efectos significativo. En ambas situaciones los poderes predictivos superan el $80 \%$, caracterizando modelos con óptimas propiedades explicativas. 
Se debe indicar que en la segunda etapa, las variables que caracterizan las condiciones de entrada y las útiles para la anticipación, son el sexo, año de egreso y grupo de dependencia. Esta información permite especificar el sector de aplicación de las intervenciones, por ejemplo colegios municipalizados son los que predominan en valores altos de sobre-duración. Por otro lado, la caracterización del año de egreso de enseñanza media como una variable de efecto significativo en la predicción de la sobre-duración invita a entender este fenómeno como generacional, disminuyendo los tiempos de sobre-duración en aquellos alumnos de promociones más nuevas. En la segunda etapa, la raíz jerárquica juega un papel fundamental, pues muchas de las variables que se indican como de primer orden o de efecto significativo directo en el tiempo de sobre-duración son ajenas al control de la universidad, por tanto, el desafío es como perturbarlas de manera indirecta, justificando el segundo y tercer orden en la raíz.

\section{Formulación matemática de los modelos}

En esta sección se explicitarán las formulaciones matemáticas del modelo, de tal manera de poder visualizar los pesos respectivos y realizar estimaciones de la sobre-duración según dimensión.

Es importante indicar las características generales de un modelo Poisson. Si la variable aleatoria $Y$ posee distribución Poisson, será indicada como: $Y \sim P_{o}(\theta)$, donde $\theta$ caracteriza el parámetro o característica poblacional necesaria para la determinación de cualquier probabilidad bajo este modelo. Una característica de suma importancia de este modelo, es el hecho de que su valor esperado o esperanza, es exactamente el parámetro $\theta$, esto es, $E(Y)=\theta$.

Para la determinación de probabilidades bajo este modelo, es usada la siguiente función de cuantía de probabilidad:

$$
P(Y=y)=\frac{\theta^{y} e^{-\theta}}{y !}, y=0,1,2, \ldots
$$

Indicar que la sobre-duración posee una distribución Poisson, es establecer que $\left[\theta^{y} e^{-\theta}\right] / y$ ! gobierna el comportamiento de los datos, además indicar que se trata de una regresión Poisson, es asumir que el parámetro $\theta$ puede ser relacionado con un conjunto de predictores, esto es $\theta=e^{B W}$, donde $W$ caracteriza la matriz de diseño o de variables independientes de estudio y $\mathrm{B}$ representa vectores de parámetros, caracterizando los pesos de cada variable independiente individual. En W se encuentran todas las variables especificadas en la raíz jerárquica de tercer orden para la sobre-duración, en función de estas especificaciones la formulación matemática para cada una de las dimensiones especificadas es:

\section{Dimensión Personal}

$$
\theta=e^{-0.87(C-0.02(U-0.15 X))-0.97(V-1.72(W+0.02 U-0.03 Z)+0.11(Y+0.2 D))-0.83(A B+0.02(Z-0.11 X))}
$$

\section{Dimensión Académica Inicial}

$$
\theta=e^{-0.8(I-0.05(H-0.004 E))}
$$

\section{Dimensión Académica Final}

$$
\theta=e^{0.44 K+0.44 O+0.48 P}
$$

\section{Dimensión Docencia}

$$
\theta=e^{-0.85(S+2(T-0.02 A H))-0.98(A K-0.26 A H-0.02 A I-0.13 A J)}
$$

Para determinar probabilidades de ocurrencia de algún evento particular, como por ejemplo la probabilidad de que un determinado alumno con ciertas características tenga un tiempo de sobre-duración de 1 año, basta con sustituir las variables observadas en los modelos anteriores y luego reemplazar en la fórmula $\mathrm{P}(\mathrm{Y}=1)=\left[\theta^{1} \mathrm{e}^{-\theta}\right] / 1$ ! , utilizando el valor de $\theta$ encontrado. Se observa que al presentar la formulación matemática del modelo, se puede visualizar el sentido anidado de las variables en segunda y tercera jerarquía. 


\section{CONCLUSIONES}

A través del presente estudio, se pudo llegar a las siguientes conclusiones: (1) Los factores que intervienen en el fenómeno de la sobre-duración, están agrupados en cuatro dimensiones, a saber: Dimensión personal, dimensión académica inicial, dimensión académica final y dimensión docencia, todas ellas concordantes con las investigaciones en Chile y Latinoamérica. (2) La organización de las variables predictoras, a través de la Raíz Jerárquica, facilita el proceso de explicitar los modelos matemáticos asociados a la estimación de la sobre-duración en cada una de las cuatro dimensiones, lo que permite implementar una política institucional de alerta temprana que permita intervenir con acciones remediales.

\section{AGRADECIMIENTOS}

Los autores agradecen al programa de Doctorado en Política y Gestión Educativa, Dirección General de Investigación (DGI CNE 06-1718) y a la Vicerrectoría de Desarrollo (UPA 1498), todas ellas pertenecientes a la Universidad de Playa Ancha, Valparaíso Chile.

\section{REFERENCIAS}

Carvajal, M.C, González, C.J. y Sarzoza, S.J., Variables sociodemográficas y académicas explicativas de la deserción en la Facultad de Ciencias Naturales de la Universidad de Playa Ancha. Revista Formación Universitaria, 11(2), en prensa (2018)

Di Gresia, L., Rendimiento académico universitario. Documento de la Universidad Nacional de la Plata. Recuperado de: https://goo.gl/ULexBP (2007)

Garbanzo, V.G., Factores asociados al rendimiento académico en estudiantes universitarios, una reflexión desde la calidad de la educación superior pública. Revista Educación, 31 (1), 43-63 (2007)

Giovagnoli, P.I., Determinantes de la deserción y graduación universitaria; Tesis Doctoral, Facultad de Ciencias Económicas, Universidad Nacional de Mar del Plata, Argentina (2001)

González, C.J., Carvajal, M.C y Viveros, R.F., Coeficientes edumétricos para la validez y dificultad de un test: Propuesta; Estudios pedagógicos (Valdivia), 42(3), 467-481 (2016)

González, G. B., Análisis de los factores que determinan el rendimiento académico de los estudiantes universitarios. Revista científica sinapsis, 1(4) (2017)

Hammond, F., Abandono y rezago estudiantil en universidades de gestión estatal: el caso de la Universidad Nacional de Mar del Plata, Tesis Doctoral, Universidad Nacional de Mar del Plata, Argentina (2016)

Hernández, S.R., Fernández, C.C y Baptista, L.P., Metodología de la investigación; Sexta Edición; Editorial Mc Graw Hill; México (2014)

Himmel, E., Modelos de análisis de la deserción estudiantil en la educación superior; Calidad de la Educación, (17), 91 107 (2002)

International Institute for Higher Education in Latin America, Informe sobre la educación superior en América Latina y el Caribe 2000-2005: La Metamorfosis de la Educación Superior, IESALC (2006)

OCDE, La Educación Superior en Chile, Ministerio de Educación, Gobierno de Chile (2009)

Oriol, G. X., Mendoza. L. M., Covarrubias, C. G y Molina. V. M., Emociones positivas, apoyo a la autonomía y rendimiento de estudiantes universitarios: el papel mediador del compromiso académico y la autoeficacia. Revista de Psicodidáctica, 22(1), 45-53 (2017)

Pérez, G.A., La eficiencia terminal en programas de licenciatura y su relación con la calidad educativa, REICE, Revista Electrónica Iberoamericana sobre Calidad, Eficacia y Cambio en Educación (2006)

Pey, R., Durán, F. y Jorquera, P., Informe para la toma de decisiones sobre duración de las carreras de pregrado en el $\mathrm{CRUCH}$, Consejo de Rectores de las Universidades Chilenas (CRUCH), Santiago, Chile (2012)

Rodríguez, S., Fita, E. y Torrado, M., El rendimiento académico en la transición secundaria-universidad. Revista de educación, 334, 391-414 (2004)

Schmelkes, S., La Combinación de Estrategias cuantitativas y cualitativas en la investigación Educativa. Reflexiones a partir de tres estudios, Revista Electrónica de Investigación Educativa, 3(2), (2001)

SIES, Informe de duración real y sobreduración de las carreras o programas generación titulados 2011-2015, https://goo.gl/vvMfdV; Servicio de información de Educación Superior, Ministerio de Educación, Santiago, Chile (2016)

SIES, Informe matrícula 2016 Educación Superior en Chile, Santiago de Chile, MINEDUC. Disponible en https://goo.gl/eNHmNz (2016)

Vera, J. Á., Ramos, D. Y., Sotelo, M. A., Echeverría, S., Serrano, D. M y Vales, J. J., Factores asociados al rezago en estudiantes de una institución de educación superior en México. Revista iberoamericana de educación superior, 3(7), 4156 (2012) 